\title{
Combined Effects of Mechanical Strain and Hydroxyapatite/Collagen Composite on Osteogenic Differentiation of Rat Bone Marrow Derived Mesenchymal Stem Cells
}

\author{
Yan Huang, ${ }^{1}$ Xufeng Niu, ${ }^{1}$ Wei Song, ${ }^{1}$ Changdong Guan, \\ Qingling Feng, ${ }^{2}$ and Yubo Fan ${ }^{1}$ \\ ${ }^{1}$ Key Laboratory for Biomechanics and Mechanobiology of Ministry of Education, \\ School of Biological Science and Medical Engineering, Beihang University, Haidian District, Beijing 100191, China \\ ${ }^{2}$ State Key Laboratory of New Ceramic and Fine Processing, Department of Materials Science and Engineering, \\ Tsinghua University, Beijing 100084, China
}

Correspondence should be addressed to Yubo Fan; yubofan@buaa.edu.cn

Received 14 June 2013; Accepted 26 July 2013

Academic Editor: Xiaoming Li

Copyright (C) 2013 Yan Huang et al. This is an open access article distributed under the Creative Commons Attribution License, which permits unrestricted use, distribution, and reproduction in any medium, provided the original work is properly cited.

\begin{abstract}
Mesenchymal stem cells (MSCs) represent a promising source for bone repair and regeneration. Recent lines of evidence have shown that appropriate strain could regulate the osteogenic differentiation of MSCs. Our previous study demonstrated that hydroxyapatite/collagen $(\mathrm{HA} / \mathrm{Col}$ ) composite also played an important role in the osteogenic differentiation of MSCs. The aim of this study is to investigate the effects of mechanical strain and $\mathrm{HA} / \mathrm{Col}$ composite on the osteogenic differentiation of rat bone marrow derived MSCs (rBMSCs) in vitro. rBMSCs were treated with cyclic strain generated by a self-designed stretching device with or without the presence of $\mathrm{HA} / \mathrm{Col}$ composite. Osteogenic differentiation levels were evaluated using reverse transcription polymerase chain reaction (RT-PCR), alkaline phosphatase spectrophotometry, and western blotting. The results demonstrated that mechanical strain combined with HA/Col composite could obviously induce the differentiation of rBMSCs into osteoblasts, which had a better effect than only mechanical strain or HA/Col composite treatment. This provides a new avenue for mechanistic studies of stem cell differentiation and a novel approach to obtain more committed differentiated cells.
\end{abstract}

\section{Introduction}

Tissue engineering is a possible alternative to current treatments for large bone defects or injuries caused by trauma or tumor $[1,2]$. Recently, mesenchymal stem cells (MSCs) have received extensive attention in the field of tissue engineering because they can be easily isolated from bone marrow, induce little immune response, have marked self-renewal properties, and possess the biological capability to differentiate into osteogenic, adipogenic, and chondrogenic lineages $[3,4]$. The application of MSCs to bone tissue engineering requires inducing in vitro differentiation of these cells into bone forming cells, osteoblasts [5].
MSCs can differentiate into osteoblasts in response to multiple environmental factors. For example, specific combinations of soluble factors including dexamethasone, ascorbic acid, and $\beta$-glycerophosphate disodium have been shown to induce osteoblastogenesis of MSCs $[3,6]$. A variety of factors, like bone morphogenetic protein (BMP) and basic fibroblast growth factor (bFGF), can upregulate expression of osteogenic related genes in MSCs [7]. Besides chemical revulsants, physical factors such as mechanical strain $[8,9]$, shear stress $[10,11]$, and compressive stress $[12,13]$ also play important roles in the osteogenic differentiation of MSCs. In addition, along with the extensive use of implantable and interventional medical devices, implanting material has 
TABLE 1: Primers for RT-PCR.

\begin{tabular}{lrr}
\hline Gene & Forward primer & Reverse primer \\
\hline ALP & $5^{\prime}$-CATGTTCCTGGGAGATGGTA-3' & $5^{\prime}$-GTGTTGTACGTCTTGGAGAGA-3' \\
cbfa1 & $5^{\prime}$-GCCGGGAATGATGAGAACTA-3' & $5^{\prime}$-GGACCGTCCACTGTCACTTT-3' $^{\prime}$ \\
COL1A1 & $5^{\prime}$-TTACTACCGGGCCGATGA-3 & $5^{\prime}$-CTGCGGATGTTCTCAATCTG-3' $^{\prime}$ \\
GAPDH & $5^{\prime}$-TGTTCCTACCCCCAATGTATCCG-3' & $5^{\prime}$-TGCTTCACCACCTTCTTGATGTCAT-3' \\
\hline
\end{tabular}

an important influence on the adaptation, remodeling, or reconstruction of tissues and cells [14-17]. Our group and other researchers have showed that hydroxyapatite/collagen (HA/Col) composite could induce the osteogenic differentiation of rat bone marrow derived MSCs (rBMSCs) [18-20]. These studies suggest an interesting biomaterial approach to affect the osteogenic differentiation of MSCs.

Since the in vivo microenvironment of bones is composed of many factors, it is necessary to consider the joint use of different factors on the osteogenic differentiation of MSCs. For instance, the in vitro mechanobiological experiments demonstrated that mechanical loadings could affect the osteogenic differentiation of MSCs cultured in soluble biochemical environment [21, 22]. Although a number of experimental and clinical studies have attempted to regenerate bones with MSCs [23-26], the combined effects of mechanical loadings and biomaterials on the osteogenic differentiation of MSCs are still unclear.

In an effort to improve the efficiency of MSC osteogenic differentiation and better understand its molecular mechanism, in this study, we investigated the effects of mechanical strain and HA/Col composite on the induction of osteoblastogenesis of rBMSCs in vitro.

\section{Materials and Methods}

2.1. Cell Culture. All experiments involving the use of 30-dayold male Sprague-Dawley rats (Peking University Laboratory Animal Center, Beijing, China) were in compliance with the Provisions and General Recommendation of Chinese Experimental Animals Administration Legislation and were approved by the Beijing Municipal Science \& Technology Commission (Permit Number: SYXK (Beijing) 2006-0025). rBMSCs were isolated from the femurs and tibias as previously described $[27,28]$. To isolate cells from bone marrow, density gradient centrifugation was performed using the percoll technique (Pharmacia, Uppsala, Sweden). rBMSCs were cultured in Dulbecco's modified Eagle medium-low glucose (DMEM-LG; Gibco, Grand Island, NY) supplemented with $10 \%$ fetal bovine serum (MDgenics, St. Louis, MO) at $37^{\circ} \mathrm{C}$ in humid air containing $5 \% \mathrm{CO}_{2}$. rBMSCs in passages $2-4$ were, divided into four groups: the control group (rBMSCs cultured with regular complete medium), the strain group (rBMSCs treated with $5 \%$ strain at a frequency of $0.5 \mathrm{~Hz}$ for $24 \mathrm{~h}$ ), the HA/Col group (rBMSCs treated with medium containing $75 \mu \mathrm{g} / \mathrm{mL} \mathrm{HA} / \mathrm{Col}$ ), and the strain $+\mathrm{HA} / \mathrm{Col}$ group (rBMSCs treated with $5 \%$ strain at a frequency of $0.5 \mathrm{~Hz}$ for $24 \mathrm{~h}$ and cultured with medium containing $75 \mu \mathrm{g} / \mathrm{mL}$
HA/Col). A self-designed mechanical stretching device was used to apply mechanical strain to cultured cells as previously described [28]. After one-day culture, F-actin filaments of cells were stained. Relative numbers of cells were evaluated by measuring the reduction of 3-(4,5-dimethylthiazol-2-yl)2,5-diphenyltetrazolium bromide (MTT) to formazan. After 7 days, the expression levels of alkaline phosphatase (ALP), core binding factor alpha 1 (cbfal), and the alpha 1 chain of type I Col (COL1A1) were assessed using reverse transcription polymerase chain reaction (RT-PCR). After 14 days, the ALP activity was detected by ALP spectrophotometry, and the expression of osteocalcin was assessed by western blotting.

2.2. Cytoskeletal Staining. Cells were fixed in $4 \%$ paraformaldehyde, then permeabilized with $0.1 \%$ Triton X-100 in PBS and blocked in $1 \%$ bovine serum albumin. Cells were incubated in Texas red isothiocyanate conjugated phalloidin (Molecular Probes, Eugene, OR) for $30 \mathrm{~min}$ to stain all F-actin filaments and with DAPI for $5 \mathrm{~min}$ to label the nuclei at room temperature. Fluorescent images were taken under a Leica TCS SPE confocal microscope (Wetzlar, Germany).

2.3. MTT Measuring. Five $\mathrm{mg} / \mathrm{mL}$ MTT was added to cells and mixed by shaking briefly on an orbital shaker. Samples were incubated for $4 \mathrm{~h}$ at $37^{\circ} \mathrm{C}$ after that process. Then, the supernatant was removed, and $150 \mu \mathrm{L}$ dimethyl sulfoxide was added following $10 \mathrm{~min}$ of oscillation. The optical density (OD) of samples was measured at $490 \mathrm{~nm}$ using a Thermo Scientific Varioskan Flash Multiplate Reader (Thermo Inc., Waltham, MA).

2.4. RT-PCR Analysis. Total RNA was extracted from cultured cells using TRIzol reagent (Invitrogen, Carlsbad, CA) according to the manufacturer's protocol and quantified using a Genequant pro RNA/DNA Calculator (Bio-Rad, Hercules, CA). cDNA was synthesized by two-step RT using the Reverse Transcriptase M-MLV (Takara, Kyoto, Japan), followed by PCR using Taq DNA Polymerase (Fermentas, Ontario, Canada). The forward and reverse sequences of the primers (synthesized by Invitrogen) in PCR are listed in Table 1. RT-PCR was performed for 30 cycles of $94^{\circ} \mathrm{C}$ for $30 \mathrm{~s}$, $55^{\circ} \mathrm{C}$ for $1.5 \mathrm{~min}$, and $68^{\circ} \mathrm{C}$ for $1 \mathrm{~min}$, with an additional $7 \mathrm{~min}$ incubation at $72^{\circ} \mathrm{C}$ after completion of the final cycle. A $10 \mu \mathrm{L}$ sample of each PCR product was size-fractionated by $1.5 \%$ agarose gel electrophoresis, and bands were visualized with a UV Transilluminator (Tanon, Shanghai, China). 


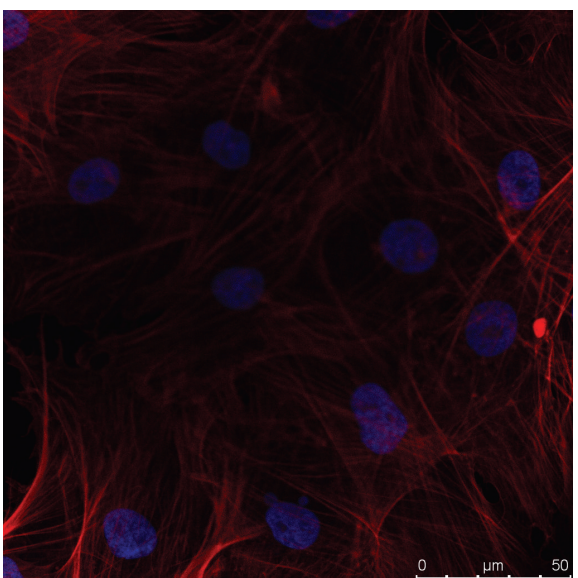

(a) Control

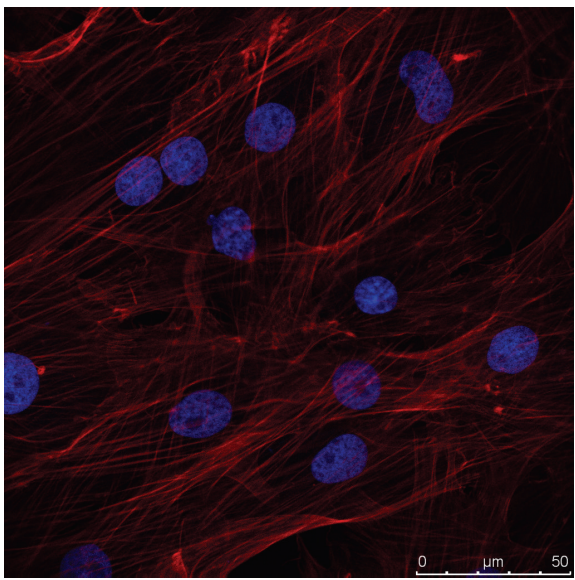

(c) $\mathrm{HA} / \mathrm{Col}$

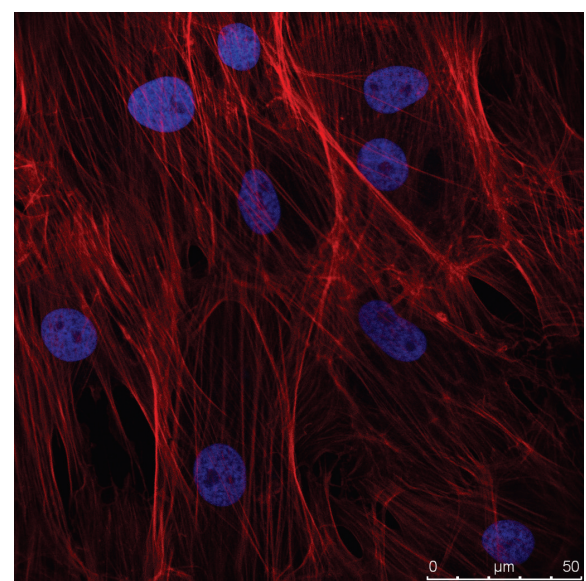

(b) Strain

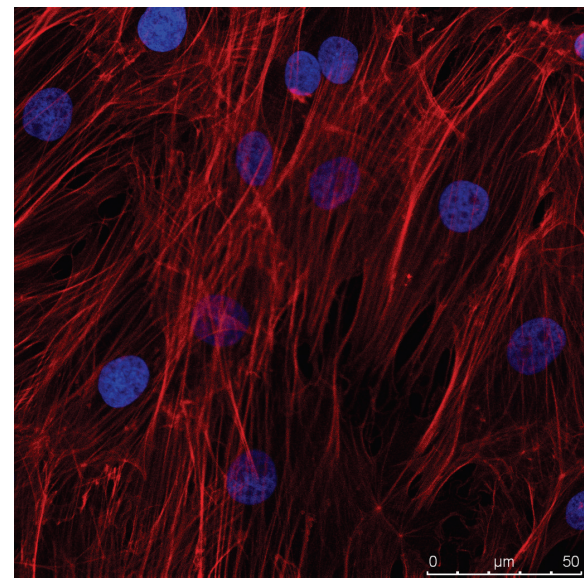

(d) Strain + HA/Col

Figure 1: F-actin cytoskeletal changes of rBMSCs exposed to mechanical strain. (a) Control; (b) strain; (c) HA/Col; (d) strain + HA/Col. rBMSCs were incubated in Texas red isothiocyanate-conjugated phalloidin to stain all F-actin filaments (red) and with DAPI to label the nuclei (blue). Mechanical strain was in left $\rightarrow$ right direction. Scale bar, $50 \mathrm{~mm}$.

2.5. ALP Quantification Assays. The ALP activity was detected by ALP spectrophotometry. The cell culture supernatants were collected, centrifuged at $2000 \mathrm{~g}$ for $10 \mathrm{~min}$ at $4^{\circ} \mathrm{C}$ to remove any debris, and analyzed for ALP using an ALP Kit (Biosino, Beijing, China) according to the manufacturer's instructions. The absorbance was read at $405 \mathrm{~nm}$ after the addition of reagents using a Thermo Scientific Varioskan Flash Multiplate Reader.

2.6. Western Blotting. Whole-cell protein extracts $(20 \mu \mathrm{g} /$ lane) were separated by SDS-PAGE and transferred to a polyvinylidene difluoride Immobilon-P membrane (Millipore, Bedford, MA) using an electroblotter (Bio-Rad). Membranes were blocked with nonfat milk (Applygen, Beijing, China) for $30 \mathrm{~min}$ at room temperature, followed by overnight incubation at $4^{\circ} \mathrm{C}$ with primary antibodies to osteocalcin (Boster, Wuhan, China) at a dilution of $1: 1000$. Primary antibody binding was detected using a HRPconjugated secondary antibody (Zhongshan Goldenbridge Biotechnology, Beijing, China) and super ECL (Applygen).
2.7. Statistical Analysis. Each experiment was conducted at least three times. All data were collected from cultures obtained from independent isolations. Statistical analysis was performed using one-way analysis of variance (ANOVA). A Turkey's test was used to determine the difference between two groups within the multiple groups. All data were expressed as mean \pm SD. Differences were considered significant when $P<0.05$. All calculations were performed using SPSS 17.0 (SPSS Inc., Chicago, IL).

\section{Results}

3.1. Effects of Mechanical Strain on F-Actin Filaments of rBMSCs. Confocal image of F-actin filaments showed that the cells cultured under static conditions had random fiber orientation. Strained rBMSCs showed filaments aligned perpendicular to the axis of mechanical strain (Figure 1).

3.2. Effects of Mechanical Strain and HA/Col Composite on the Proliferation of rBMSCs. The results of MTT analysis of 


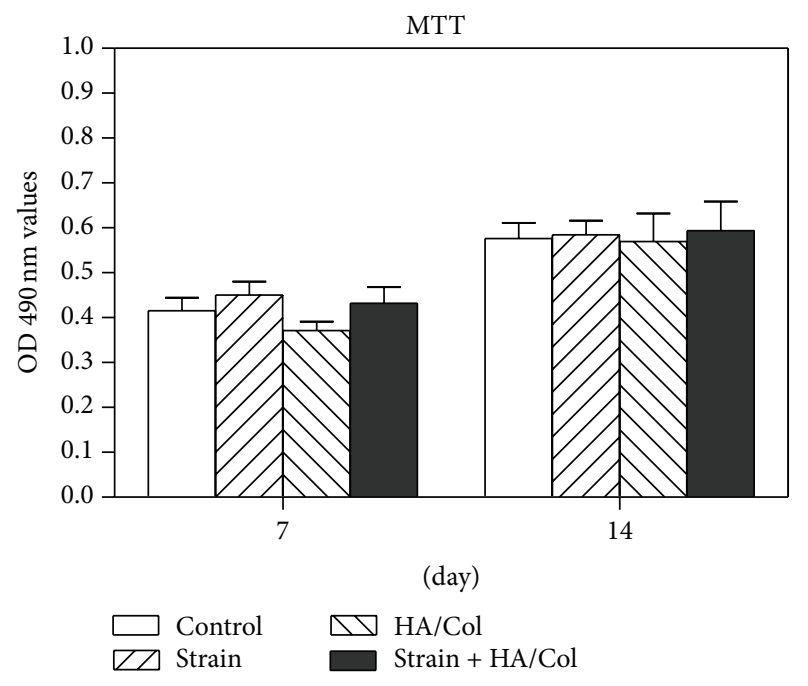

FIGURE 2: Effects of mechanical strain and HA/Col composite on the proliferation of rBMSCs measured by MTT assay.

rBMSCs in the four groups were shown in Figure 2. There were no statistical differences detected in the proliferation among the cells cultured in the four groups.

\subsection{Effects of Mechanical Strain and HA/Col Composite on the} Osteoblast-Related Gene Expressions of rBMSCs. As shown in Figure 3, both mechanical strain and HA/Col composite induced the expression of ALP, cbfal, and COL1A1, an attribute that was absent in untreated cells $(n=3, P<$ 0.05). Combination of mechanical strain and HA/Col had a stronger effect on mRNA expression of ALP, cbfal, and COL1A1 than either treatment alone $(n=3, P<0.05)$, but there was no significant difference between mechanical strain and HA/Col stimulation groups $(n=3, P>0.05)$.

\subsection{Effects of Mechanical Strain and HA/Col Composite on the} Osteoblast-Related Protein Productions of rBMSCs. To further determine the effects of mechanical strain and HA/Col composite on rBMSCs differentiation into osteoblast, the expression of ALP was assessed by ALPs pectrophotometry (Figure 4), and the expression of osteocalcin was evaluated using western blotting (Figure 5) after 14 days. In agreement with the RT-PCR results, the expressions of ALP and osteocalcin were induced by mechanical strain and/or $\mathrm{HA} / \mathrm{Col}$ composite compared to untreated cell layers $(n=3, P<$ $0.05)$, especially by the strain $+\mathrm{HA} / \mathrm{Col}$ treatment $(n=3$, $P<0.05)$.

\section{Discussion}

In this study, we put emphasis upon the role of mechanical loadings and biomaterials on the osteogenic differentiation of MSCs in vitro. The results demonstrate that mechanical strain combined with HA/Col composite can obviously induce the differentiation of rBMSCs into osteoblasts, which has a better effect than only mechanical strain or HA/Col composite treatment.

Cell osteogenic differentiation is most often judged in terms of upregulation of markers indicative of a mature, differentiated osteoblast phenotype. The results of RT-PCR analysis in this study indicate that from gene expression level mechanical strain and/or HA/Col composite can significantly promote the osteogenic differentiation of rBMSCs. cbfal, ALP, and COL1A1 are traditionally used to evaluate the osteogenic differentiation of MSCs $[9,29,30]$. There is a complete lack of osteoblast development in the animal model where the cbfal gene is knocked out $[31,32]$. ALP is an ectoenzyme involved in the degradation of inorganic pyrophosphate to provide sufficient local phosphate concentration for mineralization $[29,33]$. COL1A1 is the most abundant protein in the osteocyte environment and is osteoinductive [30]. Moreover, based on regulation of gene expression, differentiating cells express osteoblast-related proteins including ALP and osteocalcin. However, the relative numbers of cells in each group exhibit no significant differences (Figure 2), so that the increases of genes and proteins are not due to cell proliferation but the treatment of mechanical strain and/or $\mathrm{HA} / \mathrm{Col}$ composite.

The effects of HA/Col composite on the osteogenic differentiation of rBMSCs have been investigated in our previous study, and the results exhibit that the optimal concentration of $\mathrm{HA} / \mathrm{Col}$ is $75 \mu \mathrm{g} / \mathrm{mL}$ [20]. The cells of mechanical strain group in this study are subjected to a cyclic uniaxial stretch $(0.5 \mathrm{~Hz}$, $5 \%$ elongation). Koike et al. examined the effects of varying magnitudes of strain on the ST2 stromal cell line, showing that low levels of strain $(0.8 \%$ and $5 \%)$ increased ALP activity and expression of Runx2. By contrast, high levels of strain decreased ALP activity (10\% and 15\% strains ) [8]. Jagodzinski et al. also demonstrated that mechanical stimulation with both $2 \%$ and $8 \%$ elongations could promote the osteogenic differentiation of MSCs [22]. All these results indicate that mechanical strain stimulates the osteogenic differentiation of stromal cells at low magnitudes of strain.

The expression levels of osteoblast-related markers in rBMSCs significantly increase to a degree suggestive of a synergistic interaction of mechanical loadings and biomaterials. The precise mechanism for the synergistic effects is still unknown, possibly increasing secretion of factors that accelerate the osteogenic differentiation or inducing different cell populations. The cytoskeleton is affected by the extracellular microenvironment and can transduce different signals [34]. In the present study, microfilament organization changed after mechanical strain and/or HA/Col treatment(s), which might be one of the key factors in the cellular response to extracellular signals. Further investigations are needed to decipher which signaling pathways are affected by mechanical strain and/or HA/Col composite.

\section{Conclusion}

This study proves that combining mechanical strain and $\mathrm{HA} / \mathrm{Col}$ composite together has a synergistic interaction on the osteogenic differentiation of rBMSCs. By combining 


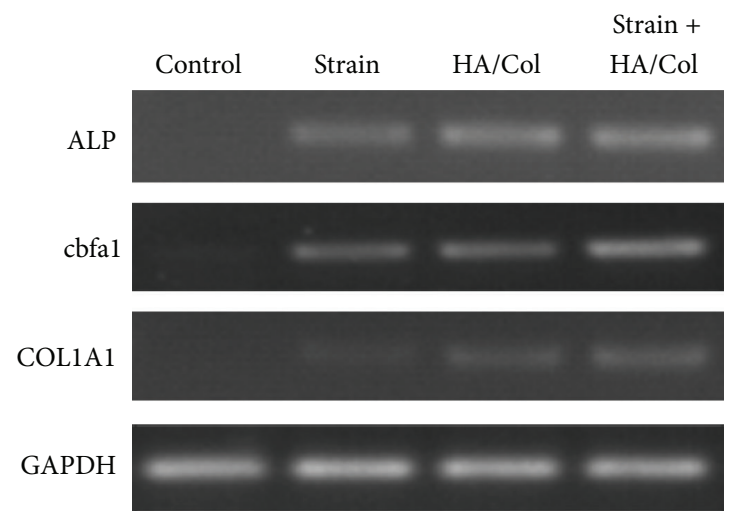

(a)
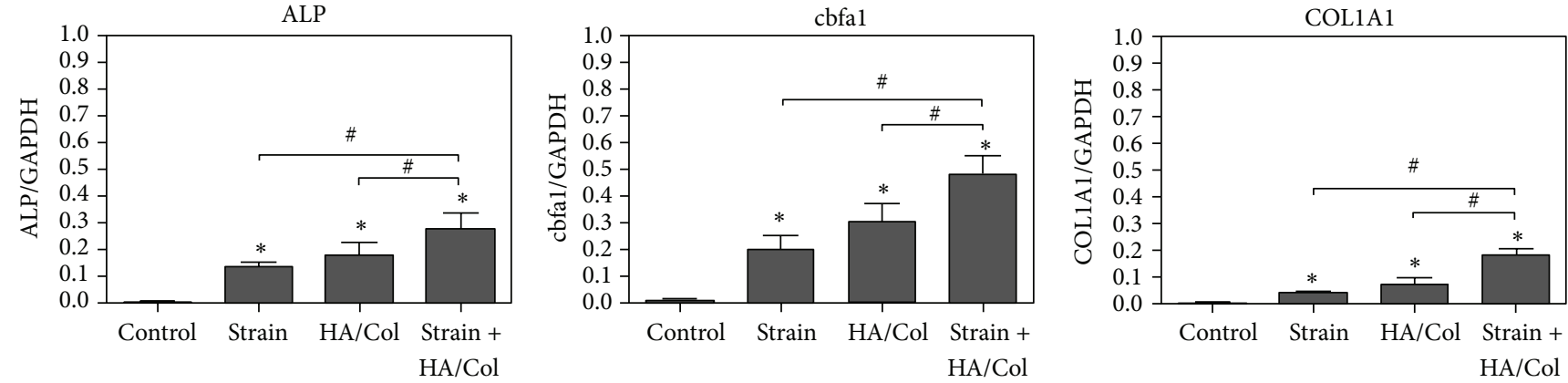

(b)

FIGURE 3: Effects of mechanical strain and HA/Col composite on the osteoblast-related gene expressions of rBMSCs. (a) Representative pictures of RT-PCR product bands. (b) Image analysis of (a). The relative expression of each gene was normalized to GAPDH. Results are shown as the mean \pm SD values $(n=3)$. ${ }^{*} P<0.05$, compared to the control group; ${ }^{\#} P<0.05$.

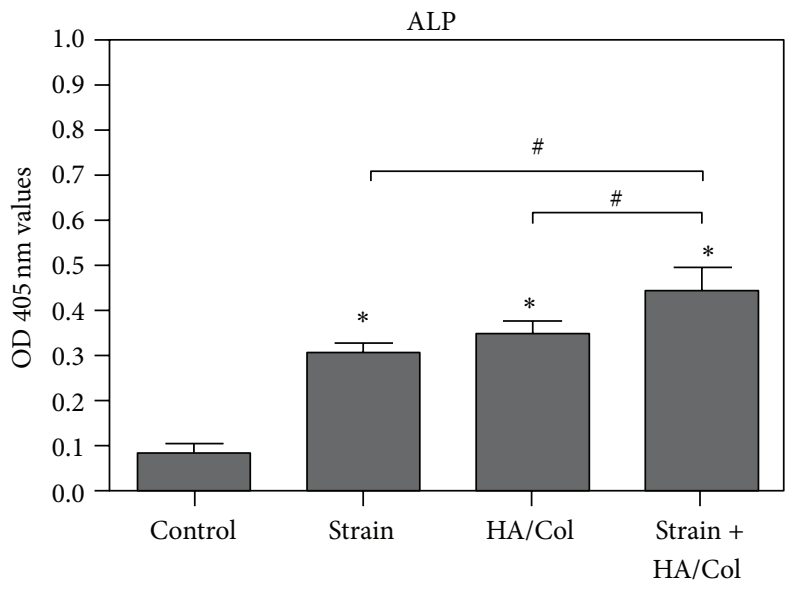

FIgURE 4: Effects of mechanical strain and HA/Col composite on the secretion of ALP into the culture media assessed by ALP spectrophotometry. Results are shown as the mean \pm SD values $(n=3) .{ }^{*} P<0.05$, compared to the control group; ${ }^{\#} P<0.05$. 


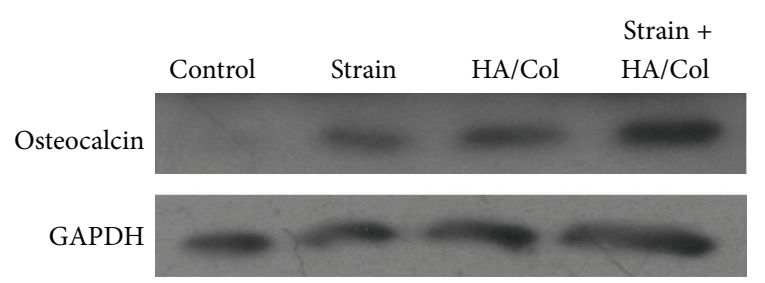

(a)

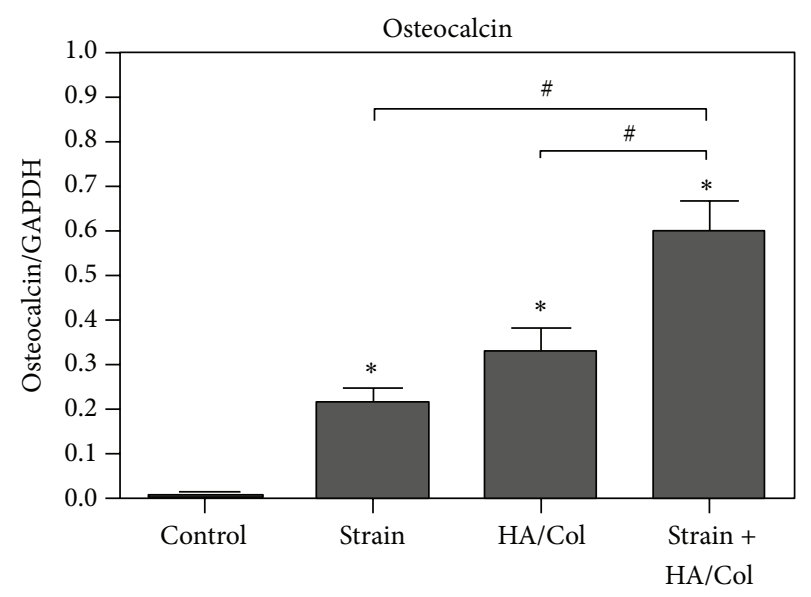

(b)

FIGURE 5: Effects of mechanical strain and HA/Col composite on the expression level of osteocalcin assessed by western blot analysis. (a) Representative pictures of western blot product bands. (b) Image analysis of (a). The relative expression of osteocalcin was normalized to GAPDH. Results are shown as the mean \pm SD values $(n=3)$. ${ }^{*} P<0.05$, compared to the control group; ${ }^{*} P<0.05$.

mechanical, biomaterial, and chemical modalities and other influential factors, one may be able to promote more rapid maturation of progenitor cells.

\section{Authors' Contribution}

Yan Huang and Xufeng Niu contributed equally to this work.

\section{Acknowledgments}

This work is supported by the National Basic Research Program of China (973 Program, no. 2011CB710901), the National Natural Science Foundation of China (nos. $11272038,11032012,11120101001$, and 10925208), the National Key Technology Research and Development Program of China (no. 2012BAI18B01), the 111 Project (no. B13003), the International Joint Research Center of Aerospace Biotechnology and Medical Engineering, Ministry of Science and Technology of China, the State Key Laboratory of New Ceramic and Fine Processing Tsinghua University, the Fundamental Research Funds for the Central Universities (nos. YWF-13-B02-007 and YWF-13-T-RSC-024), the Innovation Foundation of BUAA for PhD Graduates (no. YWF-12-RBYJ-020), and the Scholarship Award for Excellent Doctoral Student granted by Ministry of Education, China.

\section{References}

[1] D. A. Gaspar, V. Gomide, and F. J. Monteiro, "The role of perfusion bioreactors in bone tissue engineering," Biomatter, vol. 2, no. 4, pp. 167-175, 2012.

[2] X. Li, L. Wang, Y. Fan et al., "Nanostructured scaffolds for bone tissue engineering," Journal of Biomedical Materials Research A, vol. 101, no. 8, pp. 2424-2435, 2013.

[3] M. F. Pittenger, A. M. Mackay, S. C. Beck et al., "Multilineage potential of adult human mesenchymal stem cells," Science, vol. 284, no. 5411, pp. 143-147, 1999.

[4] X. Li, H. Liu, X. Niu et al., "The use of carbon nanotubes to induce osteogenic differentiation of human adipose-derived MSCs in vitro and ectopic bone formation in vivo," Biomaterials, vol. 33, no. 19, pp. 4818-4827, 2012.

[5] J. M. Seong, B. C. Kim, J. H. Park et al., "Stem cells in bone tissue engineering," Biomedical Materials, vol. 5, no. 6, Article ID 062001, 2010.

[6] A. Yamada, T. Iwata, M. Yamato et al., "Diverse functions of secreted frizzled-related proteins in the osteoblastogenesis of human multipotent mesenchymal stromal cells," Biomaterials, vol. 34, no. 13, pp. 3270-3278, 2013.

[7] N. Maegawa, K. Kawamura, M. Hirose, H. Yajima, Y. Takakura, and H. Ohgushi, "Enhancement of osteoblastic differentiation of mesenchymal stromal cells cultured by selective combination of bone morphogenetic protein-2 (BMP-2) and fibroblast growth factor-2 (FGF-2)," Journal of Tissue Engineering and Regenerative Medicine, vol. 1, no. 4, pp. 306-313, 2007.

[8] M. Koike, H. Shimokawa, Z. Kanno, K. Ohya, and K. Soma, "Effects of mechanical strain on proliferation and differentiation of bone marrow stromal cell line ST2," Journal of Bone and Mineral Metabolism, vol. 23, no. 3, pp. 219-225, 2005.

[9] M.-C. Qi, J. Hu, S.-J. Zou, H.-Q. Chen, H.-X. Zhou, and L.C. Han, "Mechanical strain induces osteogenic differentiation: Cbfal and Ets-1 expression in stretched rat mesenchymal stem cells," International Journal of Oral and Maxillofacial Surgery, vol. 37, no. 5, pp. 453-458, 2008.

[10] M. R. Kreke and A. S. Goldstein, "Hydrodynamic shear stimulates osteocalcin expression but not proliferation of bone marrow stromal cells," Tissue Engineering, vol. 10, no. 5-6, pp. 780-788, 2004.

[11] L. Liu, W. Yuan, and J. Wang, "Mechanisms for osteogenic differentiation of human mesenchymal stem cells induced by fluid shear stress," Biomechanics and Modeling in Mechanobiology, vol. 9, no. 6, pp. 659-670, 2010.

[12] S.-H. Kim, Y. R. Choi, M. S. Park et al., "Erk 1/2 activation in enhanced osteogenesis of human mesenchymal stem cells in poly(lactic-glycolic acid) by cyclic hydrostatic pressure," Journal of Biomedical Materials Research A, vol. 80, no. 4, pp. 826-836, 2007.

[13] C. Huang and R. Ogawa, "Effect of hydrostatic pressure on bone regeneration using human mesenchymal stem cells," Tissue Engineering A, vol. 18, no. 19-20, pp. 2106-2113, 2012.

[14] X. Li, C. A. van Blitterswijk, Q. Feng, F. Cui, and F. Watari, "The effect of calcium phosphate microstructure on bone-related cells in vitro," Biomaterials, vol. 29, no. 23, pp. 3306-3316, 2008.

[15] X. Li, H. Gao, M. Uo et al., "Effect of carbon nanotubes on cellular functions in vitro," Journal of Biomedical Materials Research A, vol. 91, no. 1, pp. 132-139, 2009.

[16] X. Li, Y. Yang, Y. Fan et al., "Biocomposites reinforced by fibers or tubes, as scaffolds for tissue engineering or regenerative medicine," Journal of Biomedical Materials Research A, 2013. 
[17] X. Li, Y. Huang, L. Zheng et al., "Effect of substrate stiffness on the functions of rat bone marrow and adipose tissue derived mesenchymal stem cells in vitro," Journal of Biomedical Materials Research A, 2013.

[18] K.-L. Ou, J. Wu, W.-F. T. Lai et al., "Effects of the nanostructure and nanoporosity on bioactive nanohydroxyapatite/reconstituted collagen by electrodeposition," Journal of Biomedical Materials Research A, vol. 92, no. 3, pp. 906-912, 2010.

[19] N. Hild, O. D. Schneider, D. Mohn et al., "Two-layer membranes of calcium phosphate/collagen/PLGA nanofibres: in vitro biomineralisation and osteogenic differentiation of human mesenchymal stem cells," Nanoscale, vol. 3, no. 2, pp. 401-409, 2011.

[20] Y. Huang, X. Niu, L. Wang et al., "Effects of hydroxyapatite/collagen composite on osteogenic differentiation of rat bone marrow derived mesenchymal stem cells," Journal of Composite Materials, 2013.

[21] M. E. Gomes, C. M. Bossano, C. M. Johnston, R. L. Reis, and A. G. Mikos, "In vitro localization of bone growth factors in constructs of biodegradable scaffolds seeded with marrow stromal cells and cultured in a flow perfusion bioreactor," Tissue Engineering, vol. 12, no. 1, pp. 177-188, 2006.

[22] M. Jagodzinski, M. Drescher, J. Zeichen et al., "Effects of cyclic longitudinal mechanical strain and dexamethasone on osteogenic differentiation of human bone marrow stromal cells," European Cells and Materials, vol. 7, pp. 35-41, 2004.

[23] S. P. Bruder, K. H. Kraus, V. M. Goldberg, and S. Kadiyala, "The effect of implants loaded with autologous mesenchymal stem cells on the healing of canine segmental bone defects," Journal of Bone and Joint Surgery A, vol. 80, no. 7, pp. 985-996, 1998.

[24] X. Li, Q. Feng, X. Liu, W. Dong, and F. Cui, "Collagen-based implants reinforced by chitin fibres in a goat shank bone defect model," Biomaterials, vol. 27, no. 9, pp. 1917-1923, 2006.

[25] M. Marcacci, E. Kon, V. Moukhachev et al., "Stem cells associated with macroporous bioceramics for long bone repair: 6- To 7-year outcome of a pilot clinical study," Tissue Engineering, vol. 13, no. 5, pp. 947-955, 2007.

[26] N. Hosogane, Z. Huang, B. A. Rawlins et al., "Stromal derived factor-1 regulates bone morphogenetic protein 2-induced osteogenic differentiation of primary mesenchymal stem cells," International Journal of Biochemistry and Cell Biology, vol. 42, no. 7, pp. 1132-1141, 2010.

[27] Y. Huang, X. Jia, K. Bai, X. Gong, and Y. Fan, "Effect of fluid shear stress on cardiomyogenic differentiation of rat bone marrow mesenchymal stem cells," Archives of Medical Research, vol. 41, no. 7, pp. 497-505, 2010.

[28] Y. Huang, L. Zheng, X. Gong et al., "Effect of cyclic strain on cardiomyogenic differentiation of rat bone marrow derived mesenchymal stem cells," PLoS ONE, vol. 7, no. 4, Article ID e34960, 2012.

[29] Y. Huang, X. Jin, X. Zhang et al., "In vitro and in vivo evaluation of akermanite bioceramics for bone regeneration," Biomaterials, vol. 30, no. 28, pp. 5041-5048, 2009.

[30] R. K. Schneider, A. Puellen, R. Kramann et al., "The osteogenic differentiation of adult bone marrow and perinatal umbilical mesenchymal stem cells and matrix remodelling in threedimensional collagen scaffolds," Biomaterials, vol. 31, no. 3, pp. 467-480, 2010.

[31] T. Komori, H. Yagi, S. Nomura et al., "Targeted disruption of Cbfal results in a complete lack of bone formation owing to maturational arrest of osteoblasts," Cell, vol. 89, no. 5, pp. 755764, 1997.

[32] F. Otto, A. P. Thornell, T. Crompton et al., "Cbfal, a candidate gene for cleidocranial dysplasia syndrome, is essential for osteoblast differentiation and bone development," Cell, vol. 89, no. 5, pp. 765-771, 1997.

[33] R. Marom, I. Shur, R. Solomon, and D. Benayahu, "Characterization of adhesion and differentiation markers of osteogenic marrow stromal cells," Journal of Cellular Physiology, vol. 202, no. 1, pp. 41-48, 2005.

[34] E. Tzima, "Role of small GTPases in endothelial cytoskeletal dynamics and the shear stress response," Circulation Research, vol. 98, no. 2, pp. 176-185, 2006. 

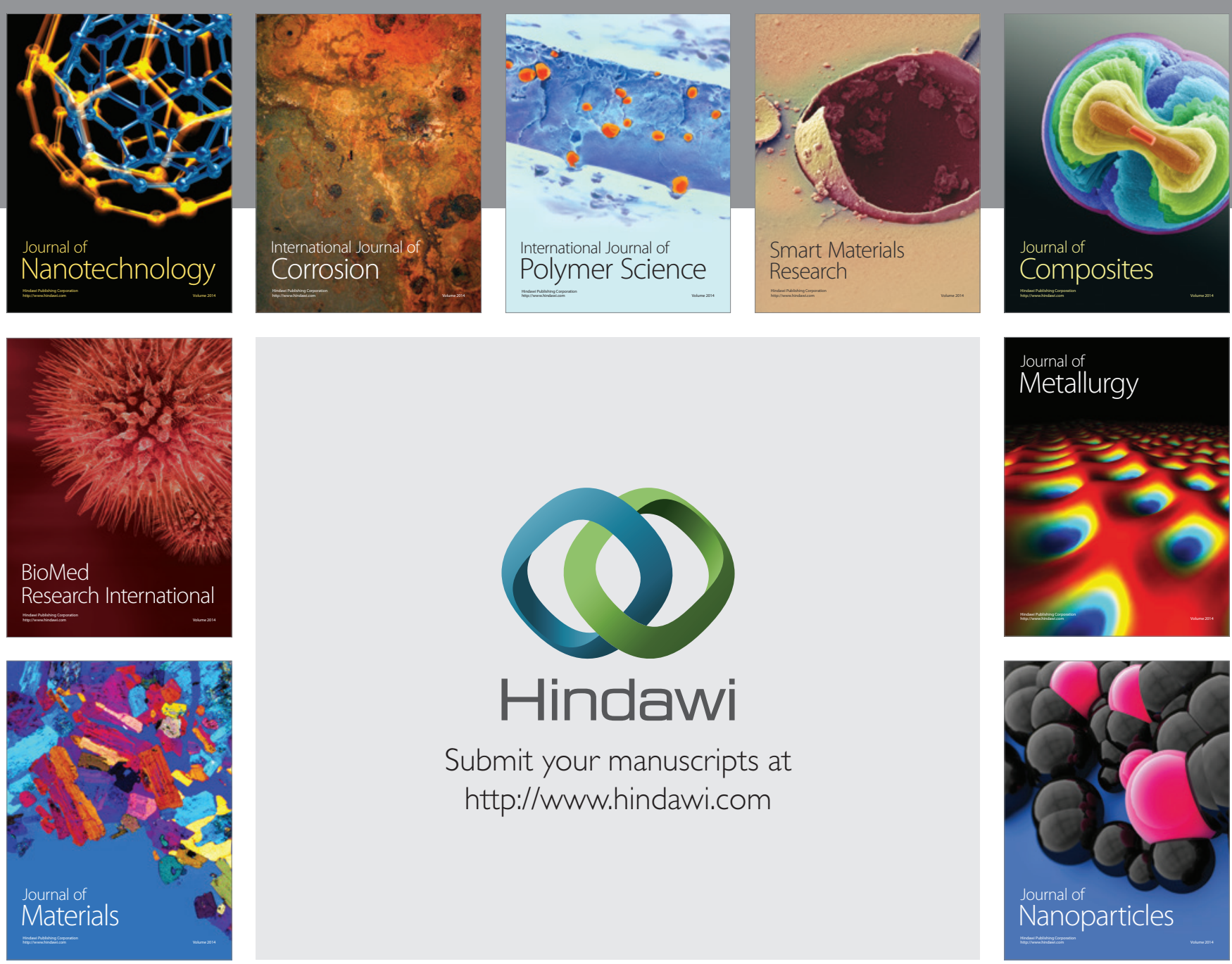

Submit your manuscripts at http://www.hindawi.com
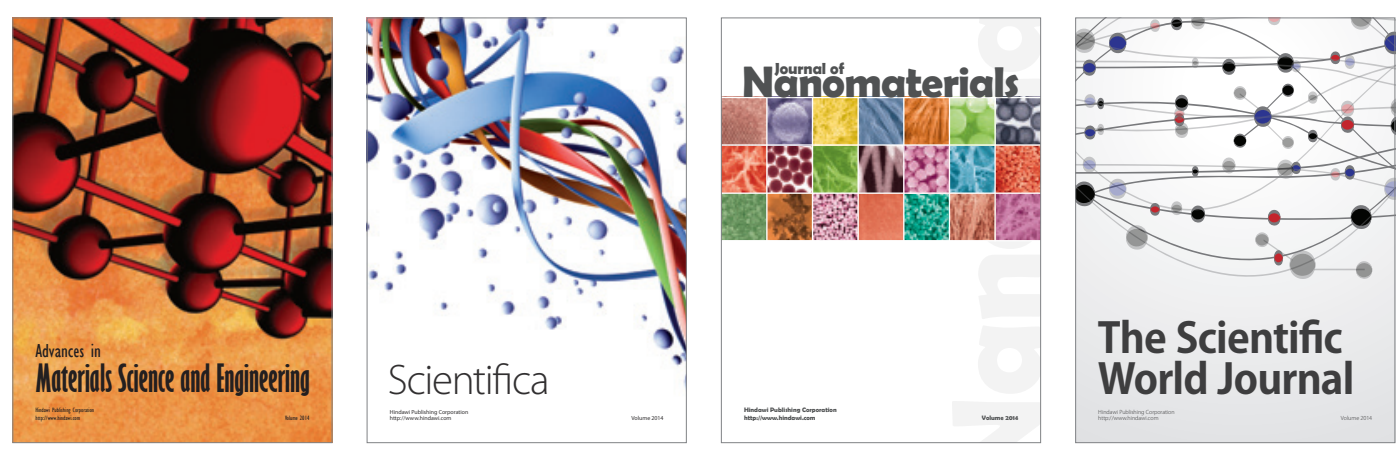

\section{The Scientific World Journal}
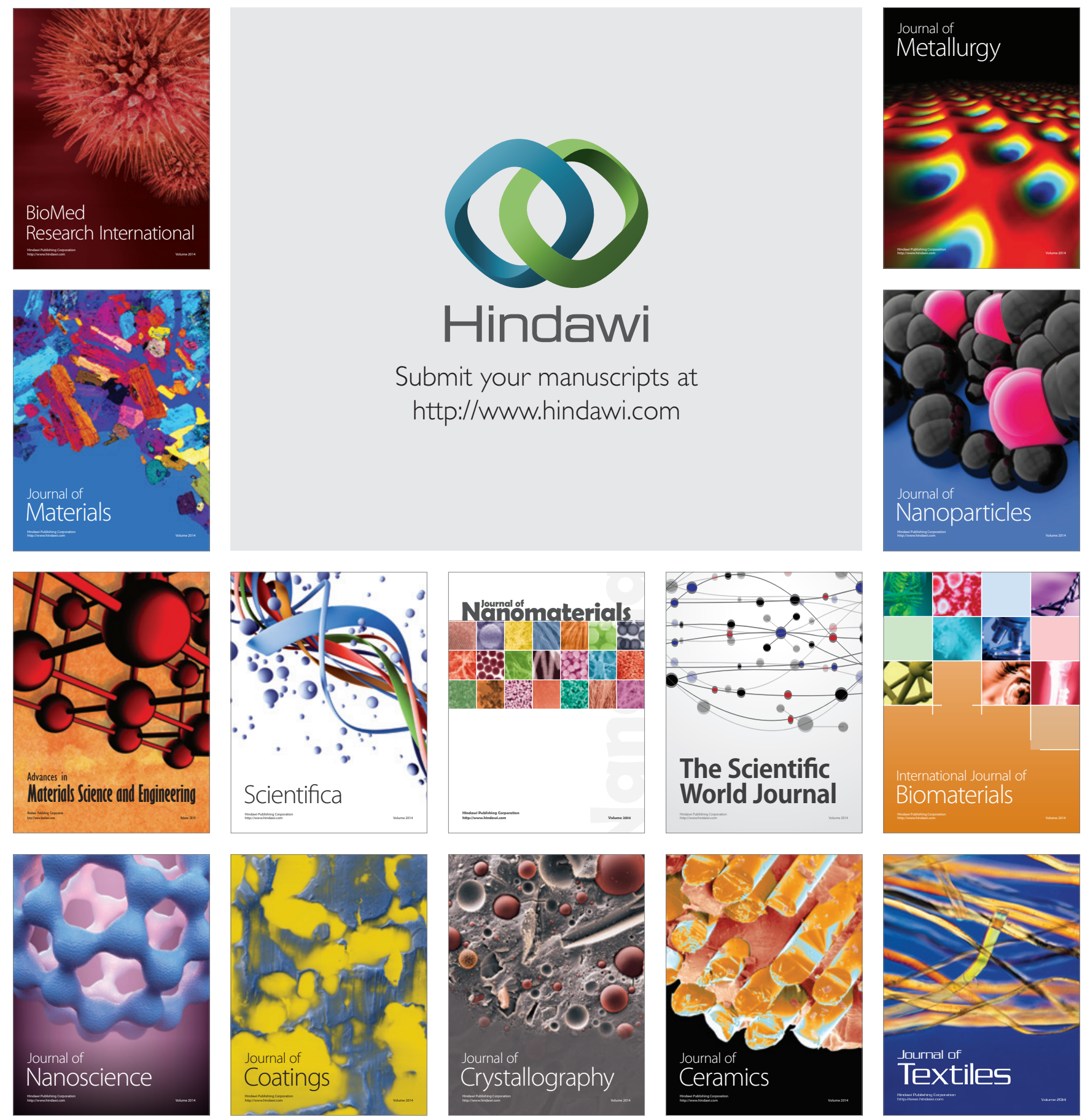